\title{
An Improved Iterative Pace-Mapping Algorithm to Detect the Origin of Premature Ventricular Contractions
}

\author{
Andony Arrieula ${ }^{1,2,3}$, Hubert Cochet $^{2,4,5,6}$, Pierre Jaïs ${ }^{2,4,5,6}$, \\ Michel Haïssaguerre ${ }^{2,4,5,6}$, Mark Potse ${ }^{3,2,1}$ \\ ${ }^{1}$ CARMEN Research Team, Inria Bordeaux Sud-Ouest, Talence, France \\ ${ }^{2}$ IHU Liryc, fondation Bordeaux Université, Pessac, France \\ ${ }^{3}$ Univ Bordeaux, IMB, UMR 5251, Talence, France \\ ${ }^{4}$ Univ Bordeaux, CRCTB, U1045, Bordeaux, France \\ ${ }^{5}$ INSERM, CRCTB, U1045, Bordeaux, France \\ ${ }^{6}$ Bordeaux University Hospital (CHU), Electrophysiology and Ablation Unit, Pessac, France
}

\begin{abstract}
Premature ventricular contraction (PVC) is one of the mechanisms that induce Ventricular Tachycardia (VT) or Ventricular Fibrillation (VF). A way to cure PVC is to ablate the origin with an endocardial catheter. But it can be difficult, and sometimes impossible, to localize this exit site accurately enough.

We propose to accelerate catheter ablation using an automatic method to guide the catheter towards the PVC origin. This method uses the QRS complex integrals of the 8-lead ECGs of the PVC and a sequence of beats paced at known locations. The method was tested using 7 realistic heart-torso models with PVCs in endocardial, epicardial, and intramural tissue.

With 10 pacing sites, $95 \%$ of the targets had been approximated to less than $5 \mathrm{~mm}$.

We conclude that although the convergence is sometimes erratic, the proposed method does converge to the origin, often within the radius of an ablation lesion.
\end{abstract}

\section{Introduction}

Premature Ventricular Contraction (PVC) is a relatively common event where the heartbeat is initiated in the ventricles rather than by the sinoatrial node. Incidental PVCs do not usually pose a danger. However, multiple PVCs can induce a ventricular tachycardia or fibrillation.

To cure very frequent and drug-resistant PVCs, the site of origin can be ablated with an endocardial catheter. To find this site, the ECG corresponding to the PVC is captured, and pacing is applied at different places to find a high correlation between the paced ECG and the spontaneous ECG. If possible the site is confirmed by waiting for a spontaneous PVC and judging, from the local electrogram, whether the catheter is at the earliest activated spot.

To move the catheter in the right direction cardiologists have to judge the differences in the ECG and translate these into directions in the heart. We propose a method that facilitates this work by computing catheter displacement advice automatically, using only the 8-lead ECG.

The method assumes that the relation between the coordinates of the PVC origin and the QRS integral of the corresponding ECG is approximately linear. This is exploited by pacing the heart at four known locations to estimate the parameters of the linear relation, which can then be used to predict the origin of the PVC. This method has been shown to work in clinical [1] and in numerical tests [2], but the localization errors are still too large to direct ablation. However, when this method is used with information from pacing sites near the origin it may become more accurate $[3,4]$. We therefore propose a new, iterative method, in which the set of known pacing sites is constantly updated with beats paced at the predicted sites.

The method works as follows: first a single PVC is captured. Then the ventricles are paced at four locations separated by several centimeters and not located on a line, and the paced ECGs are captured. In each ECG the signals are integrated over the QRS interval, to obtain an 8-element vector. Using the four paced beats a linear relation between the ECG vector and the site of origin is estimated. This relation is applied to the ectopic ECG to provide a first prediction of the site of origin. Then the ventricles are paced as close as possible to the predicted site, the paced ECG is captured, and the linear relation is re-estimated with all five pacing locations. This is repeated until the predicted location appears to converge. A flow diagram of this method was shown in previous work [5].

In this study we present a refined version of this method, 
and test it thoroughly in a set of computer models. We are in particular interested in determining 1) how many pacing sites it will take to reach a location that is sufficiently close for ablation, and 2) how often and in what situations the method will fail.

\section{Methods}

The method that we tested assumes that, for a ventricular beat originating from a single point $P$ the QRS integral of the $N$-lead ECG $(E)$ is linearly related to $P$ :

$$
P=M E+K
$$

where $M$ is a $3 \times N$ matrix and $K$ a 3 -element vector. The elements of $M$ and $K$ are fitted using a set of known $P$ and corresponding $E$.

This assumption is done to simplify a maximum the computation, while maintaining physiological consistency.

\subsection{Computational Methods}

To compute the parameters $(M, K)$, we group all the data in a single linear matrix system

$$
A \boldsymbol{\beta}=b
$$

where $\boldsymbol{\beta}$ is a $3\left(n_{l}+1\right)$-vector with $n_{l}$ the number of leads, containing the elements of $(M, K)$ to estimate, $b$ a $3 n_{p^{-}}$ vector with $n_{p}$ the number of known pacing sites, containing the $3 \mathrm{D}$ coordinates of all the pacing sites, and $A$ a $3 n_{p} \times 3\left(n_{l}+1\right)$ transfer matrix containing the QRS integrals. Then two cases arise: we have either an underdetermined system $\left(n_{l}>n_{p}+1\right)$ or an overdetermined system $\left(n_{l} \leq n_{p}+1\right)$.

To solve (2) in the underdetermined case, we use the FOcal Underdetermined System Solver (FOCUSS) method [6], which solves the following optimization problem:

$$
\left\{\begin{array}{l}
\min _{\boldsymbol{\beta}} \sum_{i}\left|\beta_{i}\right|^{p} \\
\text { subject to: } A \boldsymbol{\beta}=b
\end{array}\right.
$$

with $0<p \leq 1$. Using a Lagrange function, we obtain the iterative formula

$$
\boldsymbol{\beta}^{(k+1)}=\boldsymbol{\Pi}^{-1}\left(\boldsymbol{\beta}^{(k)}\right) A^{T}\left[A \boldsymbol{\Pi}^{-1}\left(\boldsymbol{\beta}^{(k)}\right) A^{T}\right]^{-1} b
$$

where $\boldsymbol{\Pi}(\boldsymbol{\beta})=\operatorname{diag}\left(\left|\beta_{1}\right|^{p-2}, \ldots,\left|\beta_{n}\right|^{p-2}\right)$. In this study we chose $p=1$ and $\boldsymbol{\beta}^{(0)}=(1, \ldots, 1)^{T}$.

In the case of an overdetermined system, we solve (2) minimizing the cost function

$$
J(\boldsymbol{\beta})=\frac{1}{2}\|A \boldsymbol{\beta}-b\|_{2}^{2}
$$

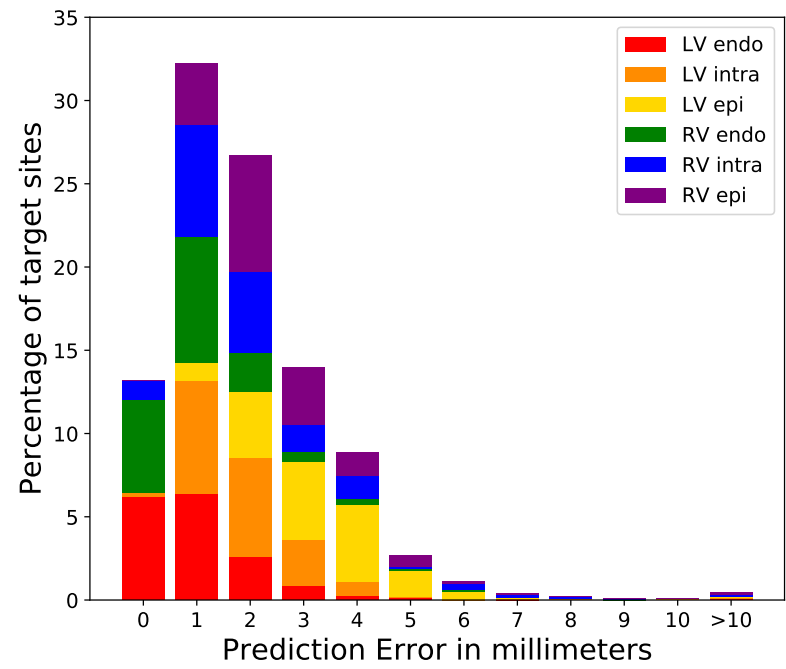

Figure 1. Euclidean distance localization error using 10 pacing sites for all the patients.

using a gradient descent method, of which the lagrangianbased iterative formula is

$$
\boldsymbol{\beta}^{(k+1)}=\boldsymbol{\beta}^{(k)}-\alpha^{(k)} A^{T} \operatorname{res}^{(k)}
$$

where the residual res ${ }^{(k)}=A \boldsymbol{\beta}^{(k)}-b$ and

$$
\alpha^{(k)}=\frac{\operatorname{res}^{(k)} \cdot A A^{T} \operatorname{res}^{(k)}}{\left\|A A^{T} \operatorname{res}^{(k)}\right\|_{2}^{2}} .
$$

In this study we chose $\boldsymbol{\beta}^{(0)}=(0, \ldots, 0)^{T}$ and $10^{6}$ iterations for the gradient descent method.

To increase the accuracy of the method, we reduce the paceable domain using the correlation between the target ECG and the pacing ECGs. For this we introduce the function

$$
f(x)= \begin{cases}2 \mathrm{~cm} & \text { if } x \in[-1,0] \\ 2\left(1-x^{2}\right) \mathrm{cm} & \text { if } x \in[0,1]\end{cases}
$$

where $x$ is the correlation between the target ECG and the considered pacing site ECG, and the output is the radius around the pacing site to remove from our eligible area.

We also chose to delete area in a $4 \mathrm{~mm}$ radius around previous pacing sites to not pace several times at the same location.

\subsection{Testing Using In-Silico Simulations}

We tested the method using detailed anatomical models of the heart and torso of 7 patients. These models were previously created from computed tomography data. Simulations were performed on 3-dimensional computational meshes, where each had a uniform edge length of $1 \mathrm{~mm}$.

For each patient, a total of 300 target PVC locations were chosen randomly in the ventricular model such that 
50 were subendocardial, 50 intramural, and 50 subepicardial in each ventricle. For each PVC the ventricular activation and 8-lead ECG were simulated.

To initiate the process, 4 initial pacing sites were chosen manually to cover the widest possible area of the considered ventricle. For LV targets the pacing sites were chosen in the LV, and for RV targets they were chosen in the RV.

We apply our method using the 8-lead ECG, so we use the FOCUSS method to make predictions until we have $8+1=9$ pacing sites in our database, and then we use the gradient descent method.

Thus, for each target PVC, we can compute a first estimation of $(M, K)$ using the FOCUSS method, so we obtain a first prediction which is projected on the paceable surface which has been reduced using (7). Then we pace at this new location and we add the resulting ECG to the database.

In practice we would repeat the process until consecutive predictions are close, but for this study we continued the process until 10 pacing sites for each target.

To simulate the ECGs, propagating action potentials were simulated with a monodomain reaction-diffusion model using the Mitchell-Schaeffer ionic model [7]. ECGs were computed using a lead-field method [8]. The simulations were performed with a recent version of the Propag-5 software and were run on a cluster computer.

\section{Results}

On average, to obtain an accuracy of less than $5 \mathrm{~mm}$, the algorithm requires 5.13 pacing sites. For the presentation and understanding of the results, we choose to present the accuracy of the algorithm when we used 10 pacing sites.

Figure 1 gives an overview of the euclidean distance localization errors divided by cardiac region. It can be seen that the vast majority of errors was smaller than $5 \mathrm{~mm}$, and less than $3 \mathrm{~mm}$ in the (sub)endocardium.

Table 1 shows the 95th percentile of the error for each patient individually. The global 95th percentile was $4.50 \mathrm{~mm}$ of error. For 6 of the 7 patients the 95th percentile was less than $5 \mathrm{~mm}$, and for patient 3 the 95th percentile was $5.67 \mathrm{~mm}$.

The accuracy for subendocardial targets was better than for subepicardial targets: we obtained a 95th percentile at $3.08 \mathrm{~mm}$ for $\mathrm{LV}$ subendocardial and $2.74 \mathrm{~mm}$ for RV subendocardial targets, versus $5.41 \mathrm{~mm}$ and $4.69 \mathrm{~mm}$ for LV and RV subepicardial targets, respectively.

Globally, there were no differences between LV and RV targets: the 95th percentile of error was $4.61 \mathrm{~mm}$ for the $\mathrm{LV}$ and $4.33 \mathrm{~mm}$ for the RV.

Table 2 shows the worst euclidean distance localization error. For patient 2, the worst prediction error was $6.22 \mathrm{~mm}$ away from target, and the target was RV intramural. For patients 4, 5, 6 and 7, the worst prediction was about $10 \mathrm{~mm}$ away from the target. These targets were intramural or subepicardial. For patient 3, the worst error was $26.34 \mathrm{~mm}$ and occurred for a target in a papillary muscle.

\section{Discussion}

We developed an iterative pace-mapping method that could be applied in the catheterization laboratory to guide an ablation catheter to the origin of a PVC, and tested it using computer models of 7 patients. We found the target PVC in $95 \%$ of the 2100 cases with an accuracy better than $5 \mathrm{~mm}$. For the other cases, the error may be due to targets being far from the paceable endocardial area, or located in anatomically complex regions.

These promising results suggest that the method can now be tested clinically. Nevertheless, further methodological improvements are desirable. Because the accuracy of the algorithm depends on the choice of the 4 initial pacing sites, the next step will be to automatically find optimal initial pacing sites. However, this choice can also be guided by the cardiologist's intuition. Indeed they can identify a likely area for the PVC and decide to pace around this area, and our method will adapt.

To simulate the 23,100 QRS complexes needed for our tests we used realistic anatomical models and ECG simulation methods but a simplified ionic model. This is not a problem because this affects only the T wave of the ECG. We verified that the QRS complex was not different from that obtained with a more detailed ionic model. In a clinical procedure, the accuracy of the algorithm can be disturbed by the patient breathing, or by the difficulty for the cardiologist to pace on the point indicated. It will be interesting to simulate a non-respect of the algorithm by the cardiologist during few iterations.

The main advantages of our method are that 1) it is fully patient-specific, while other methods used a database derived from multiple patients $[1,3]$, and 2) it does not need the heart anatomy, but only the positions of the pacing sites in $3 \mathrm{D}$ space.

The method is also able to find intramural or subepicardial PVCs using only subendocardial pacing. Thus, it can inform cardiologists on the depth of the PVC so they can adapt the strength of the ablation.

\section{Acknowledgements}

This work was supported by the Région NouvelleAquitaine, grant nr. 2017 - 1R50109 - 00013434; the European Research Council (H2020 grant agreement number 715093, ECSTATIC); and the French National Research Agency, grant references ANR-10-IAHU04-LIRYC and ANR-11-EQPX-0030. This work was granted access to 


\begin{tabular}{|l||c|c|c|c|c|c|c|c|c|}
\hline Patient & LV endo & LV intra & LV epi & LV & RV endo & RV intra & RV epi & RV & All sites \\
\hline \#1 (M,44) & 3.51 & 3.26 & 4.82 & 4.66 & 2.63 & 3.15 & 4.68 & 3.78 & 4.34 \\
\hline \#2 (F,31) & 2.72 & 2.94 & 5.02 & 4.12 & 2.05 & 3.70 & 3.97 & 3.55 & 3.93 \\
\hline \#3 (F,20) & 4.57 & 4.10 & 6.00 & 5.52 & 3.47 & 6.46 & 6.19 & 5.88 & 5.67 \\
\hline \#4 (M,59) & 3.43 & 4.02 & 5.56 & 5.21 & 3.60 & 3.74 & 4.89 & 4.67 & 4.74 \\
\hline \#5 (F,15) & 1.99 & 3.11 & 5.87 & 4.47 & 1.76 & 4.54 & 4.53 & 4.42 & 4.46 \\
\hline \#6 (M,35) & 2.24 & 2.26 & 4.19 & 3.45 & 2.23 & 5.33 & 3.55 & 4.19 & 3.90 \\
\hline \#7 (M,44) & 2.32 & 2.78 & 4.31 & 3.97 & 2.55 & 7.17 & 3.56 & 4.30 & 4.04 \\
\hline All patients & 3.08 & 3.56 & 5.41 & 4.61 & 2.74 & 4.98 & 4.69 & 4.33 & 4.50 \\
\hline
\end{tabular}

Table 1. 95th percentile of the euclidean distance localization error (in $\mathrm{mm}$ ) for each patient using 10 pacing sites.

\begin{tabular}{|c|c|c|c|c|c|c|c|c|c|}
\hline Patient & LV endo & LV intra & LV epi & LV & RV endo & $\mathrm{RV}$ intra & RV epi & $\mathrm{RV}$ & All sites \\
\hline$\# 1(\mathrm{M}, 44)$ & 4.62 & 6.77 & 5.53 & 6.77 & 3.81 & 4.53 & 16.49 & 16.49 & 16.49 \\
\hline$\# 2(\mathrm{~F}, 31)$ & 3.20 & 3.35 & 5.41 & 5.41 & 3.23 & 6.22 & 5.10 & 6.22 & 6.22 \\
\hline$\# 3(\mathrm{~F}, 20)$ & 26.34 & 15.83 & 9.56 & 26.34 & 4.22 & 26.10 & 6.61 & 26.10 & 26.34 \\
\hline$\# 4(\mathrm{M}, 59)$ & 9.95 & 4.74 & 5.60 & 9.95 & 5.51 & 4.33 & 5.14 & 5.51 & 9.95 \\
\hline$\# 5(\mathrm{~F}, 15)$ & 3.38 & 3.70 & 7.41 & 7.41 & 2.44 & 8.60 & 12.11 & 12.11 & 12.11 \\
\hline \#6 (M,35) & 2.75 & 3.48 & 5.12 & 5.12 & 6.15 & 10.82 & 4.32 & 10.82 & 10.82 \\
\hline$\# 7(\mathrm{M}, 44)$ & 3.94 & 4.03 & 11.58 & 11.58 & 3.52 & 8.87 & 11.28 & 11.28 & 11.58 \\
\hline All patients & 26.34 & 15.83 & 11.58 & 26.34 & 6.15 & 26.10 & 16.49 & 26.10 & 26.34 \\
\hline
\end{tabular}

Table 2. Worst euclidean distance localization error (in $\mathrm{mm}$ ) for each patient using 10 pacing sites.

the HPC resources of CINES under the allocation 2020A0070307379 made by GENCI.

\section{References}

[1] Sapp JL, Bar-Tal M, Howes AJ, Toma JE, El-Damaty A, Warren JW, McInnis PJ, Zhou S, Horáček BM. Real-time localization of ventricular tachycardia origin from the 12-Lead electrocardiogram. JACC Clin Electrophysiol 2017;3:687699.

[2] Kania M, Coudière Y, Cochet H, Jaïs P, Potse M. Prediction of the exit site of ventricular tachycardia based on different ECG lead systems. In Pickett C, Corsi C, Laguna P, MacLeod R (eds.), Computing in Cardiology, volume 44. Rennes, France, 2017; 253.

[3] Potse M, Linnenbank AC, Peeters HAP, SippensGroenewegen A, Grimbergen CA. Continuous localization of cardiac activation sites using a database of multichannel ECG recordings. IEEE Trans Biomed Engng May 2000; 47(5):682-689.

[4] Latour A, Grimbergen CA, Peeters HAP, Linnenbank AC, Sippens Groenewegen A. Quantitative algorithm for the localization of the origin of ectopic ventricular beats using catheter pace mapping. In Proc. 18th Annu. Int. Conf. IEEE
EMBS. 1996; .

[5] Arrieula A, Cochet H, Jaïs P, Haïssaguerre M, Potse M. Insilico evaluation of an iterative pace-mapping technique to guide catheter ablation of ventricular ectopy. In 2019 Computing in Cardiology (CinC). 2019; Page 1-Page 4.

[6] He Z, Cichocki A, Zdunek R, Xie S. Improved FOCUSS method with conjugate gradient iterations. IEEE Trans Signal Processing 2009;57:399-404.

[7] Mitchell CC, Schaeffer DG. A two-current model for the dynamics of cardiac membrane. Bull Math Biol 2003;65:767793.

[8] Potse M. Scalable and accurate ECG simulation for reactiondiffusion models of the human heart. Front Physiol 2018; 9:370.

Address for correspondence:

Andony Arrieula

CARMEN research team

Inria Bordeaux Sud-Ouest

200 Avenue de la vieille tour

33450 Talence, France

andony.arrieula@inria.fr 